\title{
A PROPÓSITO DEL DESARROLLO SOSTENIBLE: ORIGEN, EVOLUCIÓN Y TEORÍAS ALTERNATIVAS
}

\author{
Adrián Ferrandis Martínez \\ Profesor del Departamento de Geografía \\ Investigador del Instituto Interuniversitario de Desarrollo Local \\ Universitat de València \\ E-mail: adrian.ferrandis@uv.es
}

Fecha de recepción: 27/01/2016

Fecha de aceptación: 08/04/2016

\begin{abstract}
Resumen
El Desarrollo Sostenible representa el modelo de desarrollo al que todas las sociedades deberían aspirar con el objetivo de mantener en equilibrio el sistema ecológico de nuestro Planeta, al menos para conservarlo tal como hoy lo conocemos. De no ser así, las condiciones de vida existentes corren peligro de cambiar drásticamente y las consecuencias a nivel ambiental, social y económico podrían ser catastróficas. Desde la acuñación oficial del concepto, del que ya han pasado casi 30 años, los principios del Desarrollo Sostenible (a pesar de la mayor concienciación social y política) todavía quedan muy distantes de una aplicación real y de una consecución óptima de sus objetivos. Los motivos por los que se ha ralentizado esta aplicación son diversos, pero su falta de operatividad y su interpretación desarrollista por parte de los principales agentes implicados, ha dado lugar incluso al surgimiento de nuevas teorías alternativas. Es por todo esto que en este artículo se considera importante reflexionar al respecto de la redefinición del concepto, mediante la realización de un repaso a su origen y evolución, al proceso de desvirtualización de las agendas políticas para su implantación y a su confrontación con las nuevas teorías alternativas.
\end{abstract}

Palabras clave: Desarrollo Sostenible, Crecimiento Económico, Teorías Alternativas al Desarrollo Sostenible, Decrecimiento y Ecodesarrollo

\section{WITH REGARD TO SUSTAINABLE DEVELOPMENT: ORIGIN, EVOLUTION AND ALTERNATIVE THEORIES}

\section{Summary}

Sustainable development represents the model of development to which every society should aim in order to maintain balance in our planet's ecological system, at least to keep it as we know it today. Otherwise, the existing living conditions are in danger of drastically change and environmental, social and economic consequences could be catastrophic. From the official coinage 
of the concept, which have gone nearly 30 years, the principles of sustainable development (despite the increasing social and political awareness) still remain very distant of a real application and an optimal achievement of their objectives. The reasons for which this application has slowed are diverse, but their lack of operability and its developmental interpretation by key stakeholders, has led even to the emergence of new alternative theories. All this is what this article is considered important to reflect in the respect of the redefinition of the concept, by carrying out a review of its origin and evolution, the process of name of policy to its implementation agendas and their confrontation with the new alternative theories.

Key words: Sustainable development, Economic Growth, Alternative Theories to the Sustainable Development, Decrease and Eco-development.

\section{EN CE QUI CONCERNE LE DÉVELOPPEMENT DURABLE : ORIGINE, ÉVOLUTION ET ALTERNATIVE THEORIES}

Résumé.

Le développement durable représente le modèle de développement à laquelle société devrait viser afin de maintenir l'équilibre dans le système écologique de notre planète, au moins pour le garder tel que nous le connaissons aujourd'hui. Dans le cas contraire, les conditions de vie actuelles sont en danger de changer radicalement et les conséquences environnementales, sociales et économiques pourraient être catastrophiques. Depuis la reconnaissance officielle du concept, qui est passé de près de 30 ans, les principes du développement durable (en dépit de la conscience sociale et politique croissante) restent encore très éloignés d'une application réelle et une réalisation optimale de leurs objectifs. Les raisons pour lesquelles cette demande s'est ralentie sont diverses, mais leur manque d'opérabilité et de son interprétation du développement par les intervenants clés, a conduit même à l'émergence de nouvelles théories alternatives. C'est pour tout ça que dans at article on considère important reflexionner an sujet de la redéfinition de la notion, en procédant à réviser son origine et son évolution, an procès de la devirtualiser des agendas politiques pour son implantation et leur confrontation avec les nouvelles théories alternatives.

Mots-clés: Développement Durable, la Croissance Économique, les Théories Alternatives pour le Développement Durable, la Décroissement et l'Éco-développement.

\section{INTRODUCCIÓN}

El Desarrollo Sostenible es presentado por numerosos autores como el camino a seguir para salvar la Tierra de los males que la aquejan. Males causados, y sobre todo acelerados, por la acción antrópica sobre el Planeta, y en especial sobre su sistema natural o ecológico (Ramonet, 2003). La pérdida de biodiversidad, las alteraciones climáticas, la contaminación atmosférica, la intensificación de los procesos de desertización o el aumento de la pobreza humana, son algunos de esos males que la afectan y pueden llevarla a su transformación, y con ella a la modificación de las condiciones de vida que hoy conocemos.

Desde la aparición del concepto como una alternativa de desarrollo para el modelo de sociedad a la que se debería aspirar, han pasado ya casi 30 años. Sus planteamientos y objetivos permanecen todavía en un estado de aplicación muy parcial, especialmente por 
la falta de consensos y compromisos firmes (potencias como EEUU y China, se habían quedado inicialmente al margen del Protocolo de Kioto), y las reticencias en la implementación de sus vertientes más prácticas tanto desde las administraciones públicas, como desde la dirección de las grandes empresas multinacionales e instituciones internacionales.

El acuerdo alcanzado en la Cumbre del Clima de París 2015, se ha presentado como un gran acontecimiento histórico que podría marcar un antes y un después en la aplicación de medidas que favorezcan la sostenibilidad del sistema ecológico de nuestro Planeta, en especial en lo que respecta a la reducción de las emisiones de gases efecto invernadero. A pesar de ello, será necesario esperar un tiempo prudente para ratificar en qué medida los compromisos adquiridos se cumplen, aunque ya aparecen algunas dudas en directa relación con el nivel de obligatoriedad del cumplimiento de sus objetivos, que parten de compromisos de voluntariedad. Veremos en qué queda.

Al margen del acuerdo de Paris (todavía en un estado muy incipiente para su valoración desde un posicionamiento crítico y riguroso), en el presente artículo se realiza un repaso centrado en conocer cuál es el origen del concepto, qué tipo de principios lo fundamentan, cuál ha sido su evolución, qué tipo de implicaciones ha generado a nivel político, así como cuáles son las teorías que aparecen a su vez como complementarias o alternativas.

\section{EL ORIGEN DEL CONCEPTO}

A pesar de su ciertamente novedosa generalización, el concepto ya cuenta con una importante trayectoria histórica. En línea con este planteamiento, se puede decir que la palabra sostenible, que deriva del latín sus tenere, y que significa "defender", ya era utilizada en la lengua inglesa, sustainable, desde 1290 (Redclift, 2000). Etimológicamente la palabra contiene connotaciones interesantes e importantes, pues se puede referir al hecho de apoyar un estado deseado, o por el contrario, a soportar un estado no deseado. En términos generales, cuando se hace referencia a algo que es sostenible, se quiere identificar una acción o proceso que es capaz de ser mantenida/o o defendida/o. Al mismo tiempo, si se utiliza como un elemento más activo, sugiere una disposición hacia algo, que trasmite un mensaje prescriptivo de que algo hay que hacer, y que de hecho puede hacerse.

Según Gómez Orea (2007), el término sostenibilidad hace referencia a la garantía

de continuidad en el tiempo de las características estructurales y funcionales de los valores y de los atributos de aquello a lo que se refiere: sistema, recurso o actividad.

En el caso del término desarrollo, si se considera la definición del diccionario de la Real Academia Española de la Lengua, lo vincula al hecho de acrecentar o dar incremento a algo de orden físico, intelectual o moral, así como también al concepto de progresar o crecer económica, social o culturalmente.

Bermejo (2000), al hablar de los autores clásicos, indica que algunos de ellos ya tenían una visión bastante clara de la existencia de límites naturales al crecimiento ilimitado, aunque estos representaban la excepción y no la norma. De ese modo, resalta que a Malthus le preocupaba la tendencia inherente de los seres humanos a reproducirse 
ilimitadamente. A partir de esta reflexión, Malthus desarrolló la teoría de la tendencia al estancamiento del sistema capitalista desregulado, debido a que la utilización de tierras cada vez más marginales encarecía los alimentos, y en consecuencia los salarios, con lo que se deprimían los beneficios empresariales. Por su parte, de Stuart Mill destaca el hecho de que comprendía la imposibilidad de crecer ilimitadamente y abogaba por una economía de estado estacionario. Finalmente, de Marx, destaca la firme declaración que realizaba ante la incompatibilidad entre capitalismo y la preservación del equilibrio ecológico.

Con la llegada de la revolución neoclásica, desapareció cualquier tipo de preocupación por los límites físicos de la Tierra, con lo que se admitía que era una fuente inagotable de recursos y un sumidero de residuos de capacidad infinita (Bermejo, 2000).

Sin embargo, desde finales de los años 60 y principios de los 70, diferentes frentes de la sociedad, empezaron a levantar sus voces en contra de la degradación ecológica, con lo que pusieron de manifiesto el considerable impacto ambiental que estaban generando los procesos económicos productivos. Unas de estas primeras voces estuvieron encabezadas por grupos ecologistas ${ }^{1}$, en relación al uso desproporcionado de recursos naturales y la contaminación causada por los desechos de la nueva economía industrializada, que empezó a crecer a pasos agigantados a partir del final de la II Guerra Mundial. Otras organizaciones no gubernamentales (ONG) alertaban de los importantes desequilibrios económicos entre los países desarrollados y los subdesarrollados, en los que la pobreza, en lugar de erradicarse se extendía como una gran plaga.

Desde el ámbito académico y científico, pronto empezaron a aparecer intelectuales que se unieron a la línea de pensamiento de los ecologistas (en muchos casos de forma simultánea, ya que formaban parte de estos grupos), y pusieron en cuestión la viabilidad del modelo y las importantes repercusiones que este podía tener sobre el Planeta. Uno de estos precursores fue Jay Forrester, que inició los estudios previos sobre los que más adelante se daría origen al informe "Los límites del Crecimiento", solicitado por el Club de Roma (Domenech, 2007). Forrester, basándose en el análisis de sistemas, desarrolló un intento explicativo del funcionamiento del mundo, que relacionaba seis subsistemas: a) la población, b) la inversión de capital, c) el espacio geográfico, d) los recursos naturales, e) la contaminación y f) la producción de alimentos. Forrester argumentaba que el aumento de la población hacía aumentar el proceso de industrialización, lo que, a su vez, generaba una agricultura más productivista, tendente a satisfacer las crecientes necesidades alimenticias. Esa mayor cantidad de alimentos, permitía el crecimiento demográfico. Sin embargo, este autor también indicaba que esa expansión tendería a un crecimiento cero, ya que la Tierra y los recursos naturales se harían progresivamente más escasos, y el poder de regeneración del Planeta y su capacidad para absorber los residuos se resentirían considerablemente.

A partir de estos estudios previos, como se ha indicado, el primer informe que obtuvo una repercusión internacional fue el denominado "Los límites del Crecimiento", del Club de Roma, publicado en 1972 por Donella Meadows. Marcó un hito importante y

\footnotetext{
${ }^{1}$ Curiosamente en esos mismo años los activistas pacifistas, ecologistas y sus organizaciones conformaron las primeras instituciones internacionales; Friends of the Earth, Greenpeace... etc.
} 
fue el documento base sobre el que posteriormente se publicaron otros informes de referencia que oficializaron los principios del Desarrollo Sostenible (en adelante D.S.).

En este informe se desarrolló una teoría, a partir de la cual, y mediante gráficos de tendencia explicativos, alrededor del año 2050 los recursos descenderían a límites nunca vistos (Domenech, 2007). Desde un principio, este informe fue muy cuestionado, por la utilización de supuestos y extrapolaciones bastante simplistas en los que no se había tenido en cuenta la capacidad de adaptación de los seres humanos y la tecnología, en un momento en el que justamente la revolución verde demostró las posibilidades de aumentar en gran medida la producción de alimentos, por lo que estuvo desacreditado durante algún tiempo (Douglas Muschett, 1999).

A pesar de las críticas, el informe del Club de Roma, junto con la primera Conferencia de la Naciones Unidas sobre el Medio Ambiente, celebrada en Estocolmo ese mismo año, fueron unos importantes precedentes, a partir de los cuales, sobre todo de este segundo evento, se inauguró la dimensión ambiental en la agenda política internacional (Larraín, 2003). A partir de esta conferencia, las Naciones Unidas iniciaron una secuencia de conferencias y unos procesos de formalización de comisiones de investigación, que fueron los precursores en marcar las agendas oficiales de los países hacia la senda del D.S. En 1979, se celebró en Ginebra la primera Conferencia Mundial sobre el Clima, organizada por la Organización Meteorológica Mundial, el programa de las Naciones Unidas para el Medio Ambiente y el Consejo Internacional para la Ciencia. En el año 1983, diez años después de la Conferencia de las Naciones Unidas de Estocolmo, fue creada la Comisión Mundial del Medio Ambiente y el Desarrollo.

\section{La acuñación oficial del concepto}

En el año 1987, como resultado de los trabajos realizados por la Comisión Mundial del Medio Ambiente y del Desarrollo, presidida por la entonces Ministra de Medio Ambiente de Noruega, Gro Harlem Brundtland, se elaboró el informe denominado Nuestro Futuro Común, más conocido como Informe Brundtland. Sin duda alguna, este es el documento de referencia a la hora de hablar y profundizar en el conocimiento y definición del D.S., y el que le otorgó su acuñación oficial.

Esta Comisión tenía el encargo de cumplir con los siguientes objetivos: (i) Proponer unas estrategias medioambientales a largo plazo para alcanzar un D.S. en el año 2000 y a partir de esta fecha; (ii) recomendar que la preocupación por el medio ambiente pudiera traducirse en una mayor cooperación entre los países en desarrollo y entre los países que poseen diferentes niveles de desarrollo económico y social, y condujera al establecimiento de unos objetivos comunes y complementarios que tengan en cuenta la interrelación entre los hombres, los recursos, el medio ambiente y el desarrollo; (iii) examinar los cauces y medios mediante los cuales la comunidad internacional puede tratar más eficazmente los problemas relacionados con el medio ambiente; (iv) ayudar a definir las sensibilidades comunes sobre las cuestiones medioambientales a largo plazo y a realizar los esfuerzos pertinentes necesarios para resolver con éxito los problemas relacionados con la protección y mejora del medio ambiente; $(v)$ así como ayudar a elaborar un 
programa de acción a largo plazo para los próximos decenios y establecer los objetivos a los que aspira la comunidad mundial.

En una de las partes del informe se desmenuza el concepto de D.S., y se indican todas y cada una de las connotaciones y campos a los que afecta, definiéndolo, en suma, de la siguiente forma:

"El Desarrollo Sostenible es el proceso de cambio en el cual la explotación de los recursos, la orientación de la evolución tecnológica y la modificación de las instituciones, están acordes y acrecientan el potencial actual y futuro para satisfacer las necesidades y aspiraciones humanas."(Informe Brundtland, 1987).

Otra definición más sintética, es aquella que lo define como:

"La posibilidad de satisfacer las necesidades del presente sin comprometer la capacidad de las futuras generaciones para satisfacer las suyas."(Informe Brundtland, 1987).

Al final del proceso, las diferentes cuestiones acordadas se plasmaron en una serie de principios legales generales en pro de la protección del medio ambiente y el D.S. Se destacan los siguientes:

- Los derechos de todos los seres humanos a tener un medio ambiente adecuado para su salud y bienestar.

- La conservación y utilización del medio ambiente y los recursos naturales para el beneficio de las generaciones presentes y futuras.

- El mantenimiento de los ecosistemas y los procesos ecológicos indispensables para el funcionamiento de la biosfera y la conservación de la diversidad biológica.

- La incorporación del concepto de "óptimo rendimiento sostenible" en la utilización de los recursos naturales vivos y de los ecosistemas.

- El requerimiento de evaluaciones previas de las actividades que se propongan y puedan afectar considerablemente el medio ambiente o a la utilización de recursos naturales.

- La pretensión de asegurar la conservación como parte integrante de la planificación y ejecución de las actividades de desarrollo.

- La proporción de asistencia especial a los países en desarrollo en apoyo a la protección del medio ambiente y el D.S.

- La utilización de los recursos naturales transfronterizos de manera razonable y equitativa.

- El requerimiento de una evaluación ambiental de las actividades proyectadas que pudieran tener importantes efectos transfronterizos.

En definitiva, el informe asentó las bases para la definición de una teoría alternativa a las teorías económicas predominantes, enmarcadas en el crecimiento ilimitado bajo el lema "pase lo que lo pase, el negocio continúa" (Goodland, 1997). El consumo descontrolado e intensivo de los recursos naturales había provocado una degradación de los activos naturales, por lo que, mientras el imparable crecimiento económico aumentaba, el sistema natural, que da soporte a nuestras sociedades, se empobrecía. Además, junto a estas actividades de consumo de recursos de forma ilimitada, se le sumaba otra, vinculada a la generación de residuos, como resultado de las actividades productivas de 
transformación, causantes de la contaminación del aire urbano, las aguas, así como el efecto invernadero y la disminución del ozono de las capas estratosféricas.

Brundtland planteó la necesidad de establecer una agenda, en la que se detallara un proceso de transición del modelo de Crecimiento Económico al denominado D.S. Se estableció el límite del año 2000 como fecha clave en la que debía estar implantado el nuevo modelo, caracterizado por una economía más imaginativa, con una carga muy importante de desarrollo tecnológico y sobre todo bajo el prisma de hacer un uso menos agresivo de los recursos naturales, y un control de las emisiones contaminantes. Además, ese nuevo enfoque también requeriría de un esfuerzo de remodelación de las preferencias de los consumidores, dirigidas hacia unas prácticas más benignas para el medio ambiente, y hacia una reducción de los consumos productivos.

\section{LA EVOLUCIÓN DEL CONCEPTO}

La publicación oficial del Informe Brundtland impulsó todavía más el interés de la comunidad científica en profundizar en el conocimiento de aquello que se denominaba límites del crecimiento. Preocupaba la aceleración de una serie de factores que podrían provocar, en décadas, que las condiciones de vida de nuestro Planeta cambiaran sustancialmente. Además, también consiguió que, en aquella época, un concepto tan abstracto como el D.S., se convirtiera en un objetivo, al que se sumaron la mayoría de los países del Planeta².

Entre los documentos que fueron publicándose en los años sucesivos, se pueden destacar distintos autores, por el impacto, seguimiento y difusión de sus publicaciones, que no hicieron más que reafirmar y dar soporte a la nueva teoría del crecimiento. De entre estas publicaciones, podemos destacar, por ejemplo, las de R. Goodland.

Goodland (1997) desarrolló una tesis en la que se planteaba que el mundo ya había alcanzado sus límites de crecimiento. Sus argumentaciones se centraban en valorar que el ecosistema global, fuente de todos los recursos materiales que alimentan el subsistema económico y, a su vez, sumidero de sus desechos, había alcanzado una situación límite: desbordada por el crecimiento del subsistema económico, llevada al límite de su capacidad de carga, y por tanto a la generación de una situación insostenible. Todo ello auguraba un proceso de cambio o de alteración de las condiciones de vida existentes, que podía poner en peligro el futuro de las generaciones venideras (véase la figura 1).

La constatación de Goodland se asentaba sobre 5 pruebas. En primer lugar, por la apropiación de la Biomasa por los seres humanos, como resultado de la utilización del $40 \%$ (sin considerar los océanos y otros ecosistemas acuáticos) de la producción primaria de la fotosíntesis terrestre, la invasión urbana de las tierras agrícolas, el asfaltado, la erosión del suelo y la desertización, así como por la búsqueda de alimentos por parte de la población. En segundo lugar, por el calentamiento global, generado por la acumulación de $\mathrm{CO}_{2}$, que propiciaba el aumento de las temperaturas a nivel global, aunque éste es un elemento que debería ser constatado con el paso del tiempo, dada la amplia variabilidad

\footnotetext{
${ }^{2}$ Estos acuerdos fueron ratificados a partir del Congreso Internacional denominado La Cumbre de la Tierra, celebrada en la ciudad de Río de Janeiro en 1992, lugar donde se incubó la denominada Agenda 21. Para más detalle, véase el apartado 4.
}

Terra, 2016, núm. 2, pág. 74 a 96. ISSN: 2386-9968. DOI 10.7203/terra.2.8216@ @ @ @econocimiento - NoComercial (by-nc) 
climática normal del Planeta. En tercer lugar, por la rotura del escudo de ozono, como consecuencia de los gases $\mathrm{CFC}^{\prime} \mathrm{s}^{3}$, que provoca una mayor exposición a los rayos solares y aumenta las posibilidades de sufrir problemas en la piel de las personas. En cuarto lugar, por la degradación del suelo, como consecuencia de la erosión, la salinización y la desertización. Este elemento, puede tener graves consecuencias sobre la economía alimentaria mundial, ya que puede aumentar el hambre y la pobreza. Y finalmente, en quinto lugar, por la reducción de la biodiversidad, en la que quedan incluidas todo tipos de especies (tanto vegetales, como animales). El nivel de invasión del hábitat de la vida silvestre y de la extinción de especies resulta de gran importancia, ya que es un proceso acelerado.

Figura 1. Funcionamiento del ecosistema global finito

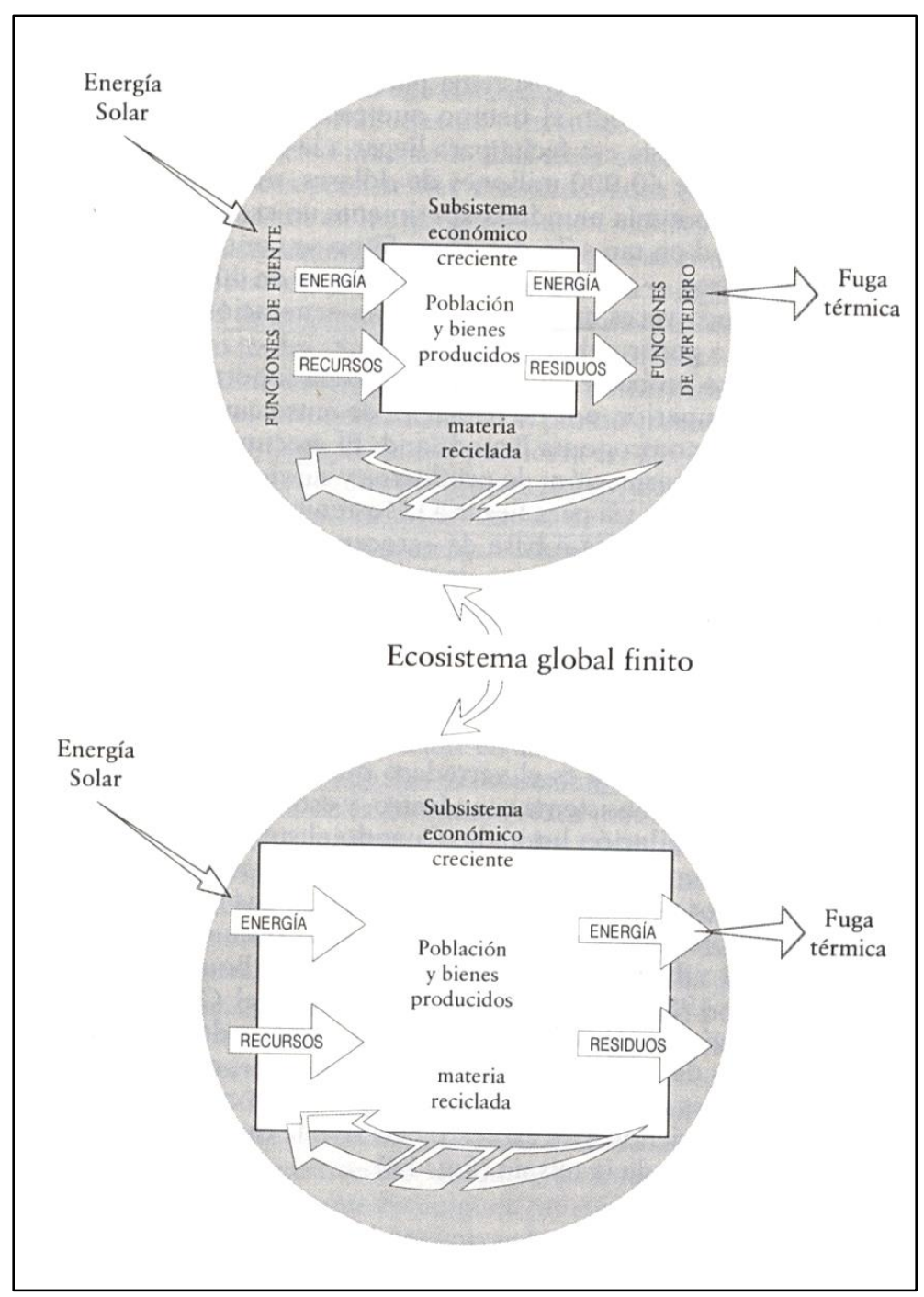

Fuente: Goodland (1997, p.21).

\footnotetext{
${ }^{3}$ Los clorofluorocarburos son derivados de los hidrocarburos saturados obtenidos mediante la sustitución de átomos de hidrógeno por átomos de flúor y/o cloro principalmente.
} 
Una vez constatado que el Planeta se encuentra en sus límites, Goodland (respaldando al Informe Brundtland) propone una serie de condicionantes que deben cumplirse, si lo que queremos es caminar en la senda del D.S. En especial identifica cuatro condiciones necesarias: 1) producir más con menos (conservación, eficiencia, mejoras tecnológicas y reciclaje); 2) reducir la explosión demográfica; 3) redistribuir a favor de los pobres el exceso de los sobreconsumidores; y 4) avanzar durante la transición del crecimiento en el uso de los recursos y en la escala de la economía hacia el desarrollo cualitativo, para que esa escala económica sea coherente con las capacidades regenerativas y asimilativas de los sistemas globales que sostienen la vida.

Otros autores continuaron desarrollando la teoría del D.S., de manera que centraron sus aportaciones en elementos específicos, como por ejemplo el papel de la inversión. Este es el caso de los científicos ${ }^{4}$ B. Von Droste y P. Dogsé (1997). Una vez argumentada la existencia de límites, y puesta en tela de juicio la máxima de que el crecimiento económico ininterrumpido no tiene por qué conducir exponencialmente hacia un mayor bienestar general, resulta evidente deducir que, para recorrer el camino que plantea la teoría del D.S., es necesaria la inversión en una serie de sectores o ámbitos determinados. Se debe invertir en un desarrollo que atienda las necesidades del presente, sin comprometer las capacidades de las generaciones futuras para atender sus propias necesidades (Informe Brundtland, 1987), en base a la relación entre la calidad medioambiental, el comportamiento de la economía y el bienestar social. Es crucial que el D.S. exija mayores inversiones en el sector del medio ambiente, para protección y restauración de la capacidad de producción y asimilación del capital natural. A pesar de ello, las inversiones no sólo han de hacerse para adaptarse a los límites que impone el ecosistema, sino también para cambiar esos límites. Por ejemplo, las inversiones que se realizan en la investigación y producción en el ámbito de la biotecnología, plantean un desafío cuyas consecuencias medioambientales y socioeconómicas tienen un gran alcance, que afecta al conjunto del Planeta. Este fenómeno, se aplica de igual modo a casos como el de las energías renovables, las tecnologías de la comunicación e incluso en la educación, la cultura y la ciencia en general.

Por su parte, Douglas Muschett (1999) se alinea con la escuela de pensamiento académico que destaca que el D.S. debe incluir una transformación radical de la sociedad. Por consiguiente, si se detallan los elementos clave que deben componer el D.S., es necesario incorporar, aparte de aspectos económicos, medioambientales y tecnológicos, algunas causas fundamentales. De este modo, según su posición, los elementos que compone el D.S. son la estabilización de la población, las nuevas tecnologías y su transferencia, la reducción de los residuos y prevención de la contaminación, las situaciones en las que todas las partes sacan provecho, la gestión integral de los sistemas medioambientales, la definición de los límites medioambientales, la depuración del concepto de la economía de mercado, la formación, los cambios en las percepciones y en las actitudes, y los cambios culturales y sociales.

Además Douglas Muschett (1999) también argumenta que no se podrá alcanzar un grado de D.S. sin contar con tecnologías mejoradas que nos permitan "estirar" los

\footnotetext{
${ }^{4}$ Expertos de la División de Ciencias Ecológicas de la UNESCO.
} 
recursos más limitados y no renovables, así como para hacer un mayor uso de los renovables. No obstante, destaca que resulta de vital importancia que, tanto los expertos en materia medioambiental como los economistas y el ciudadano de a pie, se den cuenta de que la mejora en función de la tecnología presenta igualmente sus propias limitaciones.

Por otro lado, Shen (1999), al igual que Douglas Muschett, destaca el importante papel de la aplicación de la tecnología en el aumento de la calidad de vida y en la consecución del D.S. Según nos indica, las tecnologías que son sostenibles, tanto económica como medioambientalmente, reducen la contaminación del medio ambiente de forma efectiva y suponen avances técnicos importantes con una tasa inferior de generación de residuos. Además, también contribuyen a la consecución del D.S. cuando son genéricamente aplicables a partir de sus fases iniciales de desarrollo, y ofrecen una relación favorable de rendimiento social y de rendimiento para el sector privado.

El primer criterio, relativo a la contaminación del medio ambiente, debe interpretarse con suficiente amplitud de miras como para proteger la salud, el bienestar público y los intereses ecológicos. En el segundo, se aborda la necesidad de aplicación de nuevas tecnologías, sin descartar las actuales, pero considerando que un avance técnico importante podría radicar, por ejemplo, en la utilización de fuentes de energía renovables que eliminen por completo la obligación de quemar combustible fósil. El tercero, se enmarca en el apoyo del ámbito político, para el desarrollo de las tecnologías sostenibles en el sector privado. Por último, el cuarto obedece a la gran diferencia que existe entre la rentabilidad socioeconómica y la financiación. Indica que, en ocasiones, las innovaciones tecnológicas más relevantes ofrecen un margen de beneficios sociales y económicos mucho más amplios que los que reciben los creadores.

Desde otro punto de vista, pero desde similares perspectivas, Vilches y Gil (2003) indican que, para poder sentar las bases del D.S., de tal forma que no se comprometa a las generaciones futuras, se debe poner fin a tres elementos fundamentales: a) al hiperconsumo de las sociedades más desarrolladas; b) a los desequilibrios existentes entre distintos grupos humanos, asociados a la falta de libertades y a la imposición de intereses y valores particulares, que se traducen en hambre, pobreza y marginación de amplios sectores de la población; y c) por último, a la explosión demográfica, en un Planeta con recursos limitados. Estos tres elementos son los que generan un crecimiento muy agresivo con el medio ambiente, especialmente peligroso para el conjunto de los seres vivos. Este comportamiento agresivo con el medio ambiente produce una considerable contaminación ambiental (agua, tierra y aire), una urbanización creciente, desordenada y especulativa, y un agotamiento de los recursos naturales. En definitiva implica la destrucción de la diversidad (biológica y cultural) y, en última instancia, provoca la desertificación.

Según el argumentario de estos autores, para poder contraponerse a este conjunto de problemas y desafíos, se debe imponer la universalidad de los derechos humanos, todos ellos interconectados, desde los derechos democráticos de opinión, asociación, etc., a los derechos económicos, sociales y culturales (trabajo, salud, educación...), y los derechos de tercera generación, o de solidaridad, como el derecho a un ambiente sano. Para conseguir esto, se exigen diferentes esfuerzos en la línea de: crear instituciones 
democráticas también a nivel planetario, capaces de evitar la imposición de intereses particulares nocivos para la población actual o para las generaciones futuras; impulsar una educación solidaria (que supere comportamientos orientados por valores e intereses particulares) y que contribuya a una correcta percepción de la situación del mundo; y finalmente, dirigir esfuerzos a la investigación científica, hacia el logro de conseguir tecnologías favorecedoras de un D.S.

Si se toman en consideración los principios distributivos planteados por Redclift (2000), bajo la premisa de que el D.S. es un producto del modernismo, este responde a los problemas de esta sociedad de una gran variedad de formas. De este modo, el D.S. invoca el concepto de necesidad en el contexto del desarrollo a fin de atajar los problemas de la distribución de recursos en el tiempo y el espacio. La distribución en el tiempo, entre el ahora y el luego, es decir, entre generaciones presentes y futuras, resulta esencial dentro del discurso de la sostenibilidad. La igualdad intergeneracional, en el sentido utilizado por los economistas, es la preocupación por detectar las preferencias y elecciones de las generaciones futuras aun por nacer. Esta relación, hace que tengan un gran papel en el ámbito de la economía medioambiental.

En cambio, el D.S., también responde a un problema de distribución en el espacio, la distribución de los recursos entre aquí y fuera de aquí. Se trata de los problemas de igualdad, entre sociedades diferentes, entre el Norte y el Sur. En efecto, la atención se ha centrado en los costes futuros del desarrollo para nuestras propias sociedades, como si la satisfacción de nuestras necesidades futuras fuera el principal elemento de discordancia, en lugar de serlo el modo en que actualmente se satisfacen nuestras necesidades a expensas de otros pueblos. He aquí un gran reto para la aplicación del D.S., ya que no sólo debe basarse en satisfacer las necesidades presentes y futuras de la sociedades occidentales (más desarrolladas del Norte, con algunas excepciones), sino también las del conjunto de las regiones del Planeta.

\section{Dimensiones del concepto}

Como principio general, y si se toman en consideración diferentes autores de referencia, el concepto de D.S. se clarifica, en esencia, mediante una triple dimensionalidad: la económica, la social y la ambiental. A continuación se detalla la forma de abordar esta interpretación dimensional, desde el punto de vista de algunos de esos autores.

En ese sentido, si se toma en consideración el caso de Douglas Muschett (1999), centrado en la definición de la dimensión ética del D.S., argumenta la existencia de dos vertientes: la que vincula la relación de los habitantes de un mismo país y de todo el Planeta, y la vinculante a la relación del ser humano con la Tierra y con la flora y la fauna existente en el mundo de una forma global. También resalta el valor de la ética y la cultura en el ámbito de la concepción del D.S., y destaca aspectos como el control de la natalidad o de la redistribución de la riqueza, para terminar englobándolos en la dimensión social del D.S.

Por su parte, Lee Cambell y Heck (1999) valoran la visión ecológica como una de las tres cuestiones u objetivos conceptuales clave (dimensiones), que presentan una serie de implicaciones potenciales trascendentales para el D.S. (observar figura 2). Según estos 
autores, los ecologistas suelen recalcar la conservación de la integridad de los sistemas ecológicos, que consideran cruciales para su estabilidad global a escala mundial, mediante la utilización de unidades de medida de variables físicas, químicas o biológicas. Los economistas, por su parte, tienden a primar el bienestar de los humanos con los recursos capitales y la tecnología disponible, que hace un uso de los valores económicos como unidad de medida. Mientras tanto, los sociólogos se centran en las personas, como protagonistas claves del D.S., con una serie de necesidades y anhelos aplicados a valores de medida más intangibles, como el bienestar y la delegación de atribuciones a escala social. Llegados a este punto, cabría apuntar también el papel que juegan los científicos del territorio. En especial se hace referencia a la percepción de los geógrafos, ya que la geografía es la ciencia que, bajo un enfoque holístico, global e integrador, genera capacidades marcadamente transdisciplinares, que pueden ofrecen aportaciones y soluciones más adecuadas a la compleja, variable y multidimensional tarea del D.S. (Toro, 2007; Manero, 2014).

Figura 2. Objetivos que debe integrar el Desarrollo Sostenible.

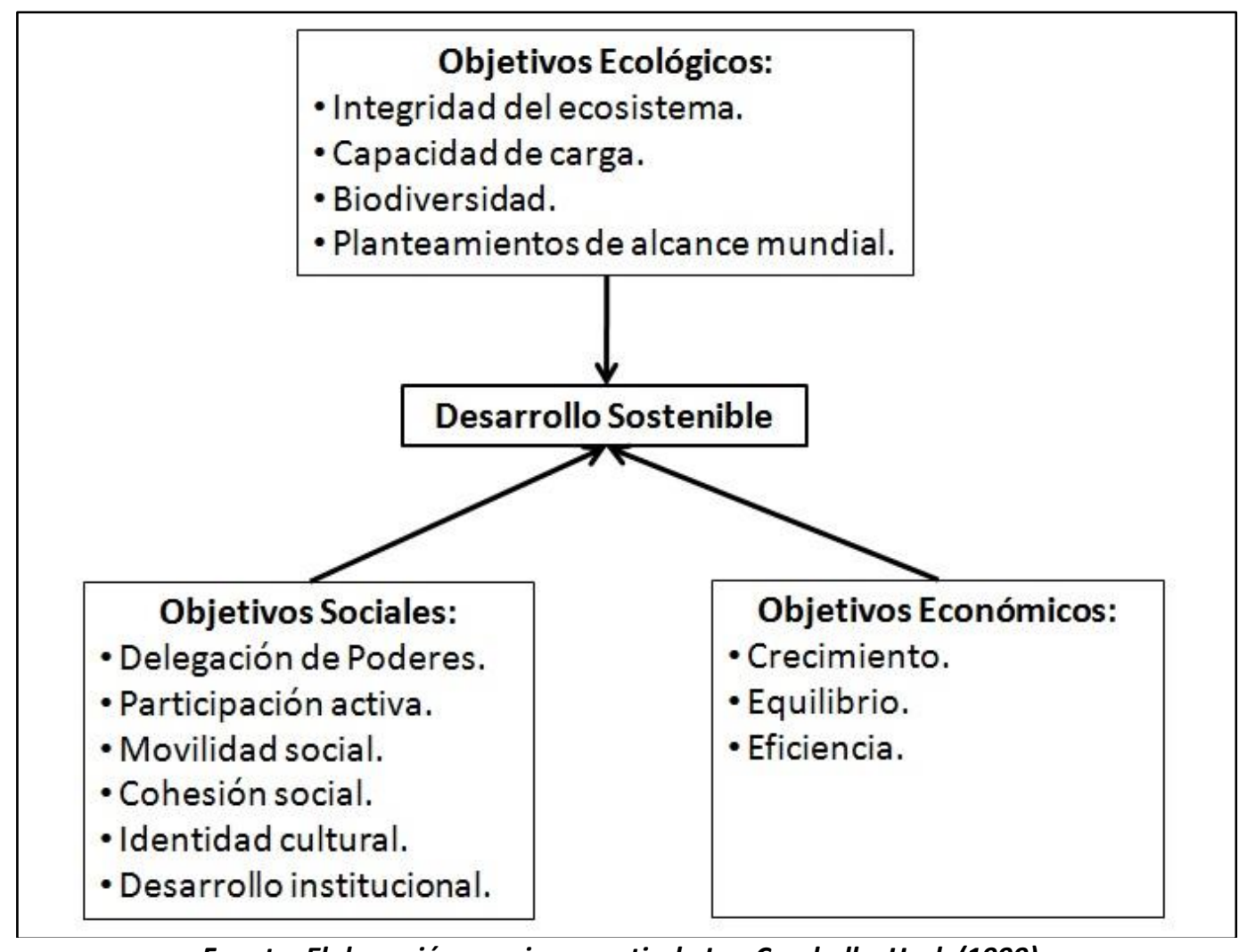

Fuente: Elaboración propia, a partir de Lee Cambell y Heck (1999).

En base a este planteamiento de objetivos, Lee Cambell y Heck (1999) consideran que las respuestas para el D.S. de los sistemas terrestres se encuentran en la intersección de las esferas que ocupan los tres ingredientes clave del D.S. (véase figura 3). El D.S., sólo se consigue cuando los objetivos de gestión y las acciones emprendidas son económica y ecológicamente viables y deseables desde el punto de vista social, de forma simultánea. 
Figura 3. Intersección de los elementos clave que engloba el Desarrollo Sostenible.

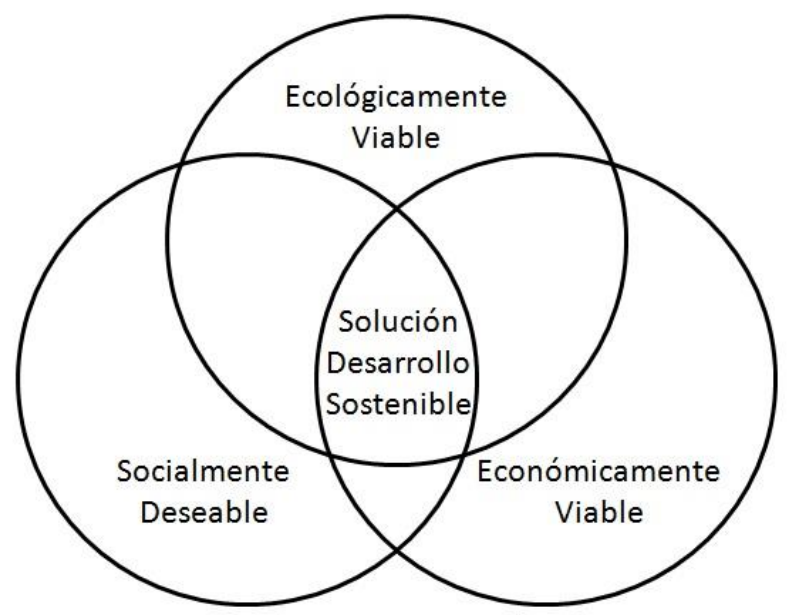

Fuente: Elaboración propia, a partir de Lee Cambell y Heck (1999).

Gómez Orea (2007), también se une a esa corriente de pensamiento, en la que se valora al D.S. desde esa triple dimensión, la económica, la social y la ambiental/ecológica. El espacio ocupado por la sostenibilidad, dentro de esa triple dimensión, es justo la zona de contacto en la que se superponen las tres a la vez. Si las actividades o líneas de crecimiento quedaran fuera de ese espacio de superposición, pasarían a situarse en las zonas del desarrollo no sostenible. Se puede argumentar que la zona de contacto representa el punto de equilibrio en el que se puede combinar el crecimiento económico, el progreso social y el respeto al medio ambiente.

Esa triple identificación, conduce a interpretar el D.S. en términos de calidad de vida y, valorada dentro del plano individual (Gómez Orea, 2007), se traduce en tres componentes: el nivel de renta, las condiciones de vida y de trabajo, y la calidad ambiental de cada individuo, junto a los que forman la sociedad. En definitiva, el concepto de D.S. se inscribe dentro de la idea optimista que considera que es compatible el desarrollo económico y la conservación del medio ambiente, o lo que es lo mismo, del medio/recurso natural sobre el que se asientan nuestras sociedades.

\section{Síntesis conceptual de la Evolución del D.S}

A continuación, y a modo de síntesis, se puede observar una tabla (figura 4) en la que quedan definidos el conjunto de principios y objetivos descritos en el conjunto de este apartado, al respecto de la evolución del concepto del D.S. 
Figura 4. Tabla de síntesis de los principios y objetivos de la evolución del concepto del D.S.

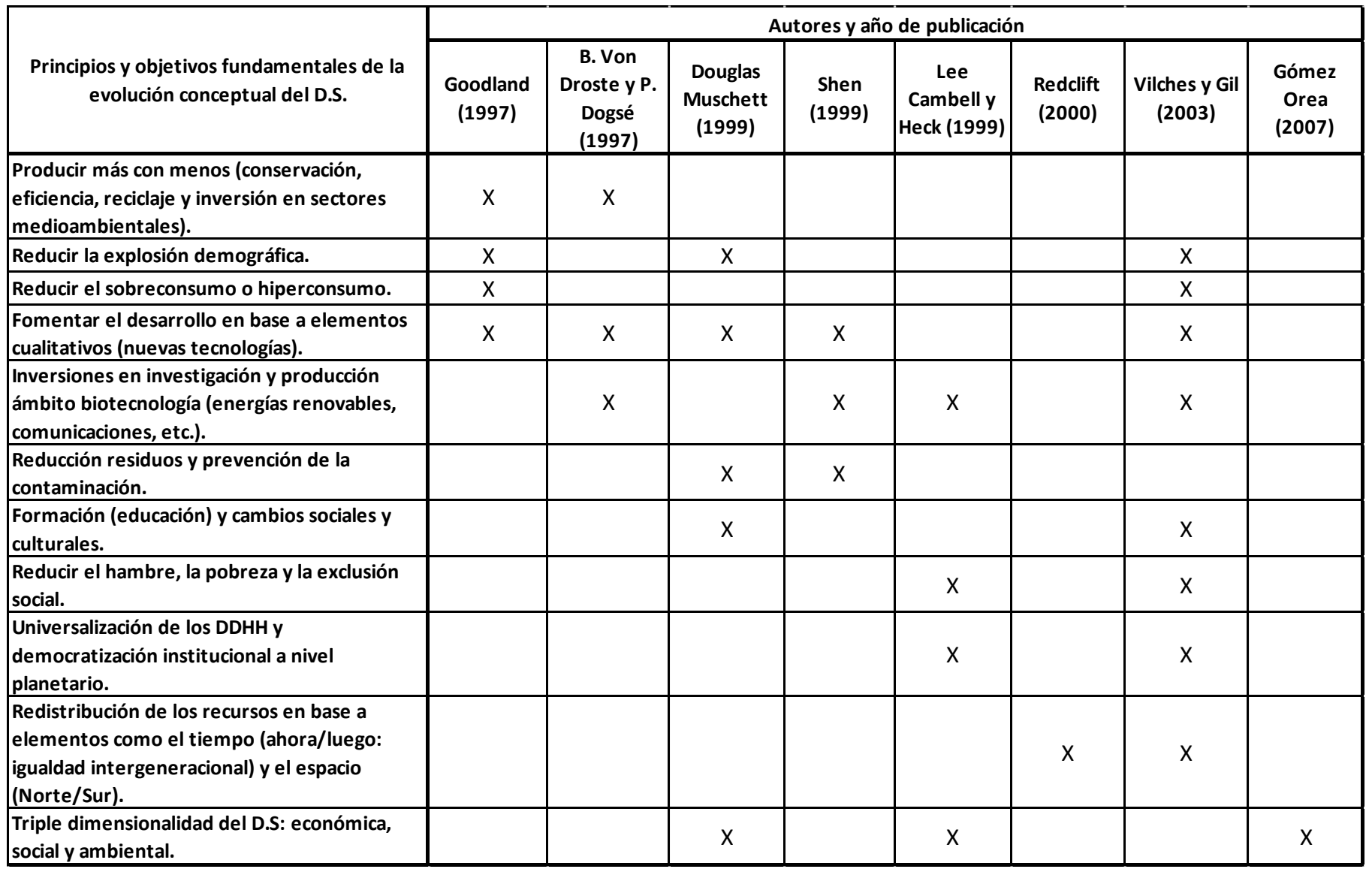

Fuente: Elaboración propia, a partir de los autores considerados y año de publicación.

\section{UNA AGENDA POLÍTICA DESVIRTUADA}

Ya se ha comentado que el Informe Brundtland fue el primer documento que estableció la necesidad de determinar una agenda política de seguimiento, que representara los intereses internacionales de caminar hacia el D.S. Este informe fue el documento central sobre el que los diferentes líderes internacionales debatieron en la llamada Cumbre de la Tierra, convocada por las Naciones Unidas, que se celebró en la Ciudad de Río de Janeiro en el año 1992. Esta cumbre tuvo lugar 20 años después de la celebración en la ciudad de Estocolmo de la reunión internacional sobre Medio Humano, en la que se habló por primera vez sobre los problemas medioambientales de la Tierra.

En la Cumbre de Río se integró el Medio Ambiente y el Desarrollo, un elemento fundamental, dadas las implicaciones dimensionales del D.S. A esta cumbre asistieron representantes de 176 estados, y 1.200 organizaciones gubernamentales y no gubernamentales, en calidad de observadores. El resultado fue un rotundo éxito, por lo menos en cuanto a intenciones declaradas, por lo que la mayoría de los países ratificaron los acuerdos allí tratados. Hubo algunas excepciones importantes, como puede ser el caso 
de los EEUU o China, precisamente los países que más contaminan. En conjunto se aprobaron 5 documentos; la Declaración de Río o Carta de la Tierra, la Agenda 21, el Convenio Sobre Diversidad Biológica, el Convenio Marco sobre el Cambio Climático y la Declaración de Principios Forestales o Declaración sobre los Bosques.

En la denominada Carta de la Tierra, quedaron definidos los principios fundamentales que debían servir de guías hacia el D.S., que ascendían a un total de 27. Entre estos se definía elementos clave, como la identificación del ser humano como centro de las preocupaciones de todo aquello que esté relacionado con el D.S., el derecho al desarrollo desde una perspectiva equitativa, que responda también a las necesidades ambientales y que considere tanto las generaciones presentes como las futuras, o la prioridad de considerar las situaciones de los países en vías de desarrollo, particularmente de los países menos adelantados y más vulnerables desde el punto de vista ambiental, entre otros (Declaración de Río, 1992).

Por su parte, la Agenda 21, partía de una idea simple, basada en considerar que el desarrollo era sostenible en la medida en la que las generaciones futuras heredaran un medio ambiente cuya calidad fuera, al menos, igual a las que recibieron las generaciones anteriores (Ramonet, 2003). Establecía la aplicación de tres principios: el principio de precaución, que favorece una aproximación preventiva, antes que reparadora; el de solidaridad entre las generaciones actuales y las futuras, y entre todas las poblaciones del mundo; y el de participación del conjunto de actores sociales en los mecanismos de decisión.

A pesar del impulso inicial que supuso la aprobación de la declaración de Río 92, la agenda política establecida pronto quedaría desvirtuada. Según nos indica Larraín (2003), durante la década de los 90 la implementación de la globalización económica neoliberal, la persistencia de la deuda externa y la reducción de la cooperación internacional, abortó cualquier posibilidad de avanzar hacia el D.S. Los procesos de evaluación de los acuerdos de Río 92 permitían constatar que la continuidad de la agenda económica impulsada por el Banco Mundial y el Fondo Monetario Internacional después de 1992, junto al incumplimiento de los compromisos de cooperación internacional ya mencionados, y los nuevos regímenes de comercialización establecidos en el marco del GATT y de la Organización Mundial del Comercio, intensificaron la destrucción ambiental y la inequidad social ya denunciados en Río 92.

Diez años después, antes de la siguiente Cumbre de la Tierra de Johannesburgo, como nos ilustran Khan, Harribey, Durand, Pengue, Rognon o Reeves, en Salvar el planeta; ecología y desarrollo sustentable (2003), lo único que se perpetuó es el crecimiento económico para algunos, mientras se agravaban, por el contrario, los problemas de inequidad social, degradación ambiental y la pérdida del poder político de los estados para dar respuesta a las necesidades de sus poblaciones.

En línea con la desvirtuación del proceso, el encuentro de Johannesburgo 2002 se caracterizó por la poca voluntad política y el escaso avance respecto a la cumbre de 1992 (Larraín, 2003). Es por este motivo, que los grandes esfuerzos, se tuvieron que centrar en salvar la Agenda de Río, la cual peligró durante todo el proceso de negociaciones, incluso desde la formulación de los mismos principios. Un ejemplo claro de esto fue, 
precisamente, la dificultad de mantener los principios de precaución, y de responsabilidades comunes pero diferenciadas, ambas consagradas en Río.

Finalmente, el Plan de Acción acordado en Johannesburgo focalizó las acciones en 6 áreas cruciales: la erradicación de la pobreza; la modificación de las modalidades insostenibles de producción y consumo; la protección y gestión de las bases de recursos naturales del desarrollo económico y social; la salud y el D.S.; y los medios de ejecución y marco institucional para el propio D.S. (Declaración de Johannesburgo, desde nuestro origen hasta el futuro, 2002).

En síntesis, a partir de Johannesburgo, se consideró la integración de los países en el mercado global como la única vía para alcanzar el D.S. (Larraín, 2003).

\section{PERSPECTIVAS ACTUALES}

A pesar de los avances realizados en algunos ámbitos de nuestros sistemas productivos (reducción parcial de emisiones, mejora de los tratamientos de depuración de vertidos, aplicación de normativas ISO para la gestión ambiental y la calidad de los procesos de fabricación, etc.) y las iniciativas de determinados estamentos de nuestras sociedades (aplicación de Agendas 21 Locales, desarrollos legislativos y normativos de medidas de evaluación de impacto de actuaciones territoriales, generación de anillos verdes en algunas ciudades, fomento parcial de la utilización de energías renovables, integración en la gestión de ciudades las técnicas de Smart Cities, etc.), la verdad es que las perspectivas actuales, aliñadas y enrarecidas por el transcurrir en un periodo de profunda crisis financiera en particular, y económica en general, de claras dimensiones globales, no animan al optimismo.

De entre las iniciativas importantes, cabe destacar la emprendida por la Unión Europea, con el grupo de ciudades denominadas Ciudades Europeas Sostenibles, firmantes de la Carta de Leipzig (2007), que establece dos grandes grupos de recomendaciones. En primer lugar, las vinculadas a hacer un mayor uso de las políticas integradas de desarrollo urbano, entendiendo por tales aquellas en las que se coordinan los aspectos espaciales, sectoriales y temporales de las áreas clave de la política urbana, como son la cohesión social y la calidad de vida, la creación y consolidación de espacios públicos de alta calidad, el impulso de la eficiencia energética, etc. En segundo lugar, se recomienda prestar especial atención a los barrios menos favorecidos dentro del contexto global de las ciudades, mediante el impulso de actuaciones integradas que conjuguen la mejora del medio ambiente físico, con el impulso a la economía y el empleo, la cohesión e inserción social y el fomento de un transporte urbano eficiente y asequible.

Por otro lado, con cierto desencanto por los resultados obtenidos, en el año 2012 fue convocada de nuevo la Cumbre de la Tierra, reunida 20 años después de su primer encuentro Mundial, justamente en la misma ciudad que le dio inicio, Río de Janeiro (Río+20).

Entre los propósitos iniciales se encontraba la idea de definir un rumbo para el Planeta que incorporara las variables medioambientales y de equidad social (la economía verde), así como buscar la forma de identificar cómo financiar la transición para los países en desarrollo o menos desarrollados (CDKN, 2012). De las principales decisiones tomadas 
destacan: la adopción de un plan decenal de nuevos estándares de producción y consumo sostenible; el lanzamiento de una negociación para establecer objetivos de D.S. (ODS); el fortalecimiento del Programa de las Naciones Unidas para el Medio Ambiente; y la decisión de crear un nuevo indicador global de riqueza que no tenga en cuenta sólo el Producto Interior Bruto (PIB) (Declaración Río+20, el futuro que queremos, 2012).

Por su parte, se identificaron, a priori, una serie de logros tangenciales: acuerdo para la protección de los océanos, que prevé la elaboración de un nuevo tratado internacional para su preservación; inicio de la negociación de un tratado para la protección de la biodiversidad en las aguas de jurisdicción internacional; o los acuerdos entre la sociedad civil y los empresarios (CDKN, 2012).

En resumen, un punto de partida, y no de llegada, caracterizado por la falta de compromisos, metas y plazos, como declaró la presidenta brasileña anfitriona de la Cumbre, Dilma Rousseff:

"Brasil fue responsable de construir un consenso posible. Ese documento es un punto de partida, no un punto de llegada. Lo que tenemos que exigir es que los países avancen a partir de ese documento" (Dilma Rousseff, en CDKN, 2012 ${ }^{5}$ ).

A partir de Río+20, se han realizado algunos avances. Una de las reuniones más destacadas (principalmente por sentar las bases del acuerdo alcanzado en la cumbre posterior de Paris 2015), fue la celebrada en septiembre de 2014 en la ciudad de Nueva York, denominada Cumbre sobre el Clima 2014: Una Acción Catalizadora. Esta cumbre estuvo organizada y alentada por las Naciones Unidas, con el objetivo de intentar aunar posturas ante la cumbre prevista para Paris 2015, en la que se deberían ratificar los acuerdos para la lucha contra el cambio climático. La versión oficial ofrecía una visión optimista de la cumbre, sobre todo por haber logrado acuerdos concretos. Entre ellos destacan el compromiso de mantener la temperatura global en menos de dos grados centígrados, mediante la reducción de emisiones, o los compromisos anunciados por empresas y gobiernos, y las coaliciones en las áreas de bosques, energía y transporte. Otro logro reseñable era el respaldo a la creación de un "Fondo Verde" para el clima, cuyo objetivo era contar con al menos 10.000 millones de dólares (ONU, 2014).

Como ya se ha indicado en el párrafo anterior y en la introducción del presente artículo, la última gran reunión internacional ha sido la celebrada en Paris 2015. Esta cumbre se denomina la Convención Marco de la Naciones Unidas sobre el Cambio Climático, XXI Conferencia de las Partes, y cuyo objetivo era el de llegar a un nuevo acuerdo internacional sobre el clima previsto para después de 2020 (una vez finalizado el periodo de implementación del conocido Protocolo de Kioto). Su principal objetivo se ha centrado en llegar a un acuerdo para limitar el aumento de la temperatura global del Planeta en menos de 20 respecto de las épocas preindustriales, y a ser posible limitar el aumento a 1,50 (ONU, 2015). Finalmente se consiguió llegar a una posición de consenso, en el llamado Acuerdo de Paris 2015, que deberá ser firmado y ratificado con los distintos países durante el periodo del 22 de abril de 2016 al 21 de abril de 2017. Este "gran

\footnotetext{
${ }^{5}$ Para más detalle véase www.cdkn.org: "Luego de Río+20: Un balance de la Conferencia de las Naciones Unidas sobre Desarrollo Sostenible. Alianza Clima y Desarrollo (2012). Última Consulta realizada en diciembre de 2015.
} 
acuerdo" nace con ciertas dudas, especialmente en lo que tiene que ver con el establecimiento de compromisos firmes y vinculantes, por lo que cabrá esperar el paso de los años para poder valorar la materialización de dichos compromisos.

\section{LAS TEORÍAS ALTERNATIVAS}

El impulso inicial que supuso la aparición de un documento como el Informe Brundtland, en el que se asentaban las bases para una nueva forma de hacer compatible el desarrollo y la mejora de la calidad de vida del conjunto de las sociedades de nuestra civilización, con la conservación del medio ambiente, quedó truncado y falto de operatividad, como consecuencia de la aprobación de unos acuerdos de carácter político que demostraban claros síntomas de inoperatividad y un marcado favoritismo en beneficio de los países más desarrollados. Una de las evidencias más claras de estas afirmaciones es el nombramiento de los organismos responsables de velar, poner en marcha y financiar las nuevas políticas que deberían guiarnos hacia ese desarrollo considerado como sostenible (la gestión económica de la Agenda 21, instrumento surgido de la cumbre de Río, fue asumida por el Fondo Mundial para el Medio Ambiente, controlado por el Banco Mundial). Todo ello, con la oposición de los movimientos ecologistas internacionales y de las ONG's, que se posicionaban junto a los representantes del grupo de los $77^{6}$, es decir de los países del Sur (Barcena, 2000).

Esta realidad se puede observar fácilmente en cualquiera de los tratados acordados en las diferentes Cumbres sobre Medio Ambiente y Desarrollo. Algunos principios allí indicados remarcan conceptos como avivar el crecimiento, o cambiar la calidad del crecimiento, que en definitiva no hacen más que remarcar la pura necesidad de crecer y crecer, vinculan desarrollo y crecimiento, y dotan al concepto de D.S. de un marcado acento desarrollista (Barcena et al., 2000), aunque resulte una versión más conservadora o respetuosa con el medio ambiente. A partir de esta situación, es cuando vuelven a aparecer voces ${ }^{7}$ que se posicionan en contra de esa interpretación errónea del D.S. Surgen nuevos conceptos o nuevas teorías, de entre las que podemos destacar las denominadas teorías del Decrecimiento o teorías del Ecodesarrollo.

La palabra decrecimiento, hasta hace relativamente pocos años, no figuraba en ningún diccionario económico o social, donde sí se podía encontrar alguna entrada sobre sus correlatos, como crecimiento cero, desarrollo sostenible y por supuesto estado estacionario (Latouche, 2008). No obstante, el término ya posee una historia relevantemente compleja y una indudable trascendencia analítica y política en el ámbito de la economía.

El economista N. Georgescu-Roegen fue uno de sus primeros defensores, y puso el énfasis en las innovaciones técnicas fundamentales que han permitido a la humanidad utilizar nuevas fuentes de energía. Desde ese punto de vista, la historia humana solo ha

\footnotetext{
${ }^{6}$ El Grupo de los 77 es el formado por el conjunto de países en vías de desarrollo, principalmente situados en el hemisferio Sur, que se unieron a partir de la cumbre de Río, para formar un frente contra los países más desarrollados, normalmente del hemisferio Norte, con el objetivo de trabajar por el D.S. desde su propia perspectiva.

${ }^{7}$ Las ONG's y las organizaciones ecologistas internacionales se han ido reuniendo de forma alternativa, en un foro global, coincidiendo con las reuniones de jefes de estado (Cumbre de Río, de Johannesburgo, etc.).
} 
conocido algunos momentos decisivos: la domesticación del fuego, la utilización de las energías fósiles y la sucesión del carbón y del petróleo. El problema de estas técnicas estriba en que acaban agotando el combustible que las hacen existir, lo que conduce a una concepción trágica de la historia de la humanidad, que está marcada por las luchas que enfrentan a los individuos y a los estados para poseer los recursos energéticos y materiales. Es uno de los pocos economistas en haber reconocido la pertinencia del primer informe del Club de Roma, y sus críticas se han limitado a aspectos menores. Sin embargo no está convencido ni por el estado estacional ni por la noción de desarrollo sostenible. Preconiza el Descrecimiento, aun siendo consciente de la necesidad primordial de mejorar las condiciones materiales de las poblaciones pobres (Urteaga, 2011).

A pesar de todo esto, existen serias dudas sobre su significado real, con lo que resulta habitual confundir regresión con decrecimiento. Según Latouche (2008), no se trata ni del estado estacionario de los viejos clásicos, ni de una forma de regresión, de recesión ni de crecimiento negativo, aunque se encuentre en él una parte del problema. Tampoco se trata de una simetría del crecimiento. La palabra en sí tiene como finalidad romper con un lenguaje estereotipado de aquellos que considera adictos al productivismo. La consigna del Decrecimiento tiene como meta insistir fuertemente en abandonar el objetivo del crecimiento por el crecimiento, cuyo motor no es otro que la búsqueda de beneficio por los poseedores de capital, y cuyas consecuencias son desastrosas para el medio ambiente. Con todo rigor, nos indica que convendría más hablar de acrecimiento, tal y como se habla de ateísmo, cuando se habla del abandono de la fe o de una religión, pero en este caso del abandono de la religión de la economía del crecimiento.

Cuando se valora la posibilidad de incorporar una nueva forma de desarrollo, basada en este caso en el Decrecimiento, rápidamente aparecen preguntas o reflexiones que intentan definir qué implicaría la aplicación de esas teorías alternativas o, al menos, de los principios que proponen. Una de esas preguntas sería; ¿Es posible mantener el índice de crecimiento sin modificar profundamente la sociedad? Como indica Latouche (2008), si estudiamos lúcidamente el problema, queda claro que la respuesta es no, por lo que se observa que no se trata de crecimiento cero, sino de crecimiento incluso bajo cero. De lo que se trata es de reducir nuestro crecimiento económico y sustituirlo por una noción basada en otra cultura de la felicidad y del bienestar, y eso pasa ineludiblemente por la necesidad de disminuir el nivel material de nuestra vida.

Si se toma como referencia lo que indica Besset (2005), pasar del infierno del crecimiento insostenible al paraíso del decrecimiento convencional supone un cambio profundo de los valores en los cuales creemos y sobre los que organizamos nuestras vidas. Por eso, para salir del imaginario dominante del crecimiento por el crecimiento, es necesario reevaluar y reconceptualizar las ideas asociadas al progreso o al progresismo, de tal forma que se reestructuren, redistribuyan y relocalicen los fundamentos del sistema imperante (Latouche, 2008).

Por su parte el denominado Ecodesarrollo, al igual que el Decrecimiento, no es una idea innovadora que parta de planteamientos recientes. A pesar de que en la actualidad retomen fuerza, ya tienen también una cierta trayectoria histórica. La noción del Ecodesarrollo fue propuesta por Strong en 1972, tras la conferencia de Estocolmo, con el 
objetivo de reactivar el diálogo entre el Norte y el Sur. Uno de los principales teóricos que ha desarrollado la propuesta de Strong, fue Sahcs ${ }^{8}$ (Urteaga, 2011), quien define las estrategias del Ecodesarrollo en base a 5 pilares fundamentales. En primer lugar, la reducción de las desigualdades, ya que según él, el crecimiento económico, incluso si es fuerte y está acompañado de una modernización de las estructuras productivas, no conduce al desarrollo, sino a incrementar las desigualdades sociales, que son responsables en buena parte del deterioro del medio ambiente. En segundo lugar, la autonomía de las decisiones: si el desarrollo sostenible aparece como una cuestión general que se plantea a todos los países, ésta debe declinarse de forma diferente según los lugares y las circunstancias. En tercer lugar, la atención prestada al desarrollo local: el desarrollo regional y local es concebido como una de las vías posibles para superar la crisis económica y medioambiental. En cuarto lugar, la elección de las técnicas apropiadas: desde la óptica del Ecodesarrollo, el análisis de las técnicas se considera como un fenómeno multidimensional, que exige tomar en consideración el tipo de energía y de recursos empleados para asegurar su funcionamiento, sus resultados, su complejidad, la calificación de la mano de obra que requiere y su impacto medioambiental, y no únicamente a través de los conceptos de capital y de trabajo. Por último, en quinto lugar, la planificación participativa, que debe ser un instrumento esencial para la prospectiva y la exploración de las opciones de desarrollo. Resulta preciso poner en marcha una planificación participativa que permita un justo equilibrio entre el mercado, el Estado y la sociedad civil (Urteaga, 2011).

\section{CONCLUSIONES}

Como se ha indicado en diferentes partes de este artículo, la aplicación del D.S. dista todavía de ser una realidad plausible y eficaz. Los principios del D.S. continúan siendo plenamente válidos e imprescindibles, con la finalidad de abordar un cambio en la forma de organización y funcionamiento de nuestras sociedades. Por tanto, su redefinición a nivel conceptual, no se considera necesaria. Caminar hacia una economía más imaginativa de base tecnológica, reducir el consumo de recursos naturales, controlar las emisiones contaminantes, remodelar la forma de consumir (en relación a las preferencias de los consumidores), junto con la reducción de los consumos productivos, continúan siendo elementos fundamentales. A todos estos elementos, se les debe sumar los objetivos de conservación, eficiencia y reutilización. Además, también resulta muy importante caminar hacia un cambio cultural y social, en especial en la forma de relacionarse con el medio natural, pero también en el modo de vida en general (sobre todo en las sociedades más desarrolladas) en el que existe una gran relación entre la mejora de la calidad de vida y el "hiperconsumo". Desconectar estos dos elementos se considera como uno de los grandes retos, que sin duda implicará cambios sustanciales. Tampoco se debe perder de vista la variable demográfica, con importantes desequilibrios a nivel continental. $Y$ en estrecha relación con este último elemento, cabe destacar igualmente la importancia de la

\footnotetext{
${ }^{8}$ Economista defensor del Ecodesarrollo. Considerado como ecosocioeconomista, por su vinculación del desarrollo con el crecimiento económico, bienestar social y preservación del medio ambiente. Ostentó diferentes cargos importantes en órganos internacionales durante más de 30 años.
} 
necesaria redistribución de la riqueza en pro de conseguir un mayor equilibrio entre las sociedades más desarrolladas y las más pobres. En definitiva generar un modelo de sociedad centrado en propiciar un desarrollo cualitativo mucho más respetuoso con los ciclos ecológicos.

Quizás, en lo que se debería poner el acento (al igual redefinir), es en la aplicación de aquello que se denomina sostenibilidad fuerte (Naredo, 1996). Es decir, traducir las implicaciones que derivan de la aplicación del D.S, avanzando en la implementación de los elementos más prácticos, de forma que puedan ser evaluables y tener un seguimiento claro sobre su evolución y sobre si realmente aportan mejoras sustanciales a nivel de grado de sostenibilidad. Esto debería ser aplicable en todas las dimensiones del D.S., y por tanto concretado para su aplicación en todos los campos del funcionamiento de nuestras sociedades, siempre bajo criterios de control y seguimiento oficialmente reconocidos y regulados. Campos como la planificación y gestión de los espacios urbanos, las necesidades de movilidad, la gestión de deshechos y aguas residuales, la ordenación y gestión de los espacios naturales, la regulación de las actividades económicas (en especial las que tienen que ver con emisiones contaminantes), las explotaciones agrícolas y ganaderas, la educación ambiental y su relación con las pautas de consumo, entre muchos otros, representan una muestra de los campos que requieren una atención especial.

Para concluir, se considera que la aparición de teorías alternativas es la consecuencia de la incapacidad de la clase política actual de liderar ese cambio necesario, de tal forma que se sobrepongan los intereses particulares de los grandes poderes económicos del Mundo y del capitalismo en general (entendido este como aquella parte del engranaje del sistema capitalista que sólo prima la vertiente económica, menospreciando la social y la ambiental), y se mire más por los interés del bien común y del Planeta en particular. Estas teorías alternativas también forman parte de diferentes formas de entender el D.S., tanto por lo que implica su aplicación, como por la óptica (Norte/Sur) desde la que se aborde. Las realidades sociales del Planeta son muy dispares y desiguales, por lo que plantean uno de los principales desafíos que el D.S. deberá afrontar para su correcta aplicación. Grandes desafíos que sin duda la capacidad humana está preparada para superar, si al final, así se lo propone.

\section{BIBLIOGRAFÍA}

Barcena, I., Ibarra, P. y Zubiaga, M. (2000): "A modo de Introducción". En Desarrollo sostenible; un concepto polémico. Serie de Ciencias Sociales de la Universidad del País Vasco, Vol. n.ㅇ, Bilbao, pp. 11-16.

Barcena, I. (2000): “¿Hacia un desarrollo sostenible?, crítica ecologista al Vo programa europeo de medio ambiente (1992-2000) y su aplicación en el Estado español”. En Desarrollo sostenible; un concepto polémico. Serie de Ciencias Sociales de la Universidad del País Vasco, Vol. n.요, Bilbao, pp. 177-230.

Bermejo, R. (2000): “Acerca de las dos visiones antagónicas de la sostenibilidad". En Desarrollo sostenible; un concepto polémico. Serie de Ciencias Sociales de la Universidad del País Vasco, Vol. n.ㅇ, Bilbao, pp. 67-103.

Terra, 2016, núm. 2, pág. 74 a 96. ISSN: 2386-9968. DOI 10.7203/terra.2.8216@@@ @ Reconocimiento- NoComercial (by-nc) 
Besset, J.P (2005): Comment ne plus être progressiste... sans devenir reáctonnaire. Paris, Fayard, $332 \mathrm{pp}$.

Brundtland, H.G. (1987): Nuestro Futuro Común o informe Brundtland. Comisión mundial del medio ambiente y del desarrollo, Naciones Unidas. Madrid, Alianza, 460 pp.

CDKN, Alianza Clima y Desarrollo (2012): "Luego de Río+20: Un balance de la Conferencia de las Naciones Unidas sobre Desarrollo Sostenible". www.cdkn.org. Consulta realizada en fecha de diciembre de 2013.

Doménech, J.L. (2007): Huella ecológica y desarrollo sostenible. Madrid, AENOR Ediciones, $398 \mathrm{pp}$.

Douglas Muschett, F. (1999): "Un método Global para el desarrollo sostenible". En Principios del Desarrollo Sostenible. Madrid, AENOR, pp. 17-63.

Douglas Muschett, F.; Lee Campbell, C.; Evans, V.; Henck, W.W.; Si Duk Lee, V.S.; Shen, T.; y Warrer, J.L. (1999): Principios del Desarrollo Sostenible. Madrid, AENOR, 207 pp.

Gómez Orea, D.: (2007): Evaluación Ambiental Estratégica; Un instrumento para integrar el medio ambiente en la elaboración de planes y programas. Madrid, Mundi-Prensa, $365 \mathrm{pp}$.

Goodland, R. (1997): "Introducción". En Medio ambiente y desarrollo sostenible. Más allá del Informe Brundtland. Colección de Estructuras y Procesos, Serie de Medio Ambiente, Madrid, Editorial Trotta, 7-19 pp.

Goodland, R.; Daly, H.; El Serafy, S.; y Von Droste, B. (Eds.) (1997): Medio ambiente y desarrollo sostenible. Más allá del Informe Brundtland. Colección de Estructuras y Procesos, Serie de Medio Ambiente, Madrid, Editorial Trotta, 133 pp.

Khan, S.A. (2003): "Desarrollo sustentable, una idea desvirtuada". En Salvar el Planeta; ecología y desarrollo sustentable. Selección de artículos publicados en Chile. Santiago de Chile, Editorial Aún Creemos en los Sueños, pp. 19-23.

Larraín, S. (2003): "Entre la vida y los negocios: la agenda política post Johannesburgo". En Salvar el Planeta; ecología y desarrollo sustentable. Selección de artículos publicados en Chile, Santiago de Chile, Editorial Aún Creemos en los Sueños, 7-14 pp.

Latouche, S. (2008): La apuesta por el decrecimiento: ¿Cómo salir del imaginario dominante?. Colección de ecología Antrazyt, n.273, Capellades (Barcelona), Editorial Icaria, $277 \mathrm{pp}$.

Lee Cambell, C. y Heck, W.W. (1999): "La perspectiva ecológica del desarrollo sostenible". En Principios del Desarrollo Sostenible. Madrid, AENOR, pp. 65-87.

Manero Miguel, M. (2014): "Desafíos e incertidumbres en torno a la Ordenación Sostenible del Territorio: Una perspectiva Euro-Latinoamericana". Boletín de la Asociación de Geógrafos Españoles, n.65, pp. 343-365.

Naciones Unidas (1992): Declaración de Río Sobre el medio Ambiente y el Desarrollo. Conferencias de Río de Janeiro.

- (2002): Desde nuestro origen hasta el futuro. Conferencias de Johannesburgo (Río+10), $190 \mathrm{pp}$.

- (2012): EL futuro que Queremos. Conferencias de Rio de Janeiro (Río+20), 60 pp. 
- (2014): "La Cumbre sobre el Clima finaliza con compromisos concretos". En http://www.un.org/climatechange/summit2014/. Consulta realizada en diciembre de 2015.

- (2015): Acuerdo de París 2015. Convención Marco sobre el Cambio Climático; Conferencia de las Partes de Paris, $40 \mathrm{pp}$.

Naredo, J.M (1996): "Sobre el origen, el uso y el contenido del término sostenible". Primer catálogo español de buenas prácticas. Madrid, Ministerio de Fomento, pp. 21-28.

Ramonet, I. (2003): "Salvar el Planeta". En Salvar el Planeta; ecología y desarrollo sustentable. Selección de artículos publicados en Chile. Santiago de Chile, Editorial Aún Creemos en los Sueños, pp. 15-18.

Redclift, M. (2000): "El desarrollo sostenible; necesidades, valores, derechos". En Desarrollo sostenible; un concepto polémico. Barcena, I., Ibarra, P. y Zubiaga, M., Serie de Ciencias Sociales de la Universidad del País Vasco Volumen n.ㅇ, Bilbao, pp.17-38.

Shen, T. (1999): "La importancia del papel de la tecnología en la consecución del desarrollo sostenible". En Principios del Desarrollo Sostenible. Madrid, AENOR, pp. 88-119.

Toro Sánchez, F.J. (2007): "El Desarrollo Sostenible: Un concepto de interés para la Geografía". Cuadernos Geográficos, n.40, pp. 149-181.

Urteaga, E. (2011): "Las teorías alternativas del desarrollo sostenible". Boletín de la Asociación de Geógrafos Españoles, n.55, pp. 113-126.

Vilches, A. y Gil, D. (2003): Construyamos un futuro sostenible; Diálogos de supervivencia. Colección Ciencia, Tecnología, Sociedad e Innovación, Cambridge University Press en colaboración con la Organización de Estados Iberoamericanos, Madrid, 275 pp.

Von Droste, B. y Dogsé, P. (1997): "El desarrollo Sostenible. El papel de la inversión". En Medio ambiente y desarrollo sostenible. Más allá del Informe Brundtland. Colección de Estructuras y Procesos, Serie de Medio Ambiente, Madrid, Editorial Trotta, pp. 87-99. 